\title{
Benzodiazepine Use and Morbidity-Mortality Outcomes in a Geriatric Palliative Care Unit: A Retrospective Review
}

\author{
Helen Senderovich a, b, c, d Sandra Gardner ${ }^{a, e}$ Anna Beralla, e \\ Michael Ganion b, c, ${ }^{\text {Dennis Zhang }}{ }^{g}$ Danusha Vinoraj ${ }^{\text {h }}$ Sarah Waicus ${ }^{i}$ \\ aBaycrest, Faculty of Medicine, Toronto, ON, Canada; ${ }^{\mathrm{b}}$ University of Toronto, Toronto, ON, Canada; ${ }^{\mathrm{c} D e p a r t m e n t}$ \\ of Family and Community Medicine, Toronto, ON, Canada; 'Division of Palliative Care, Toronto, ON, Canada; \\ 'Biostatistics Division, Baycrest, Kunin-Lunenfeld Centre for Applied Research and Evaluation (KL-CARE), Dalla \\ Lana School of Public Health, University of Toronto, Toronto, ON, Canada; ${ }^{\mathrm{B} B e t h e l l ~ H o s p i c e, ~ T o r o n t o, ~ O N, ~ C a n a d a ; ~}$ \\ 9Department of Family Practice, University of British Columbia, Vancouver, BC, Canada; hFaculty of Medicine, \\ University of Ottawa, Ottawa, ON, Canada; 'School of Medicine, Trinity College Dublin, Dublin, Ireland
}

\section{Keywords}

Benzodiazepines · Delirium · Palliative care $\cdot$ Hospice $\cdot$

Geriatrics - Terminal care

\begin{abstract}
Introduction: Patients often experience delirium at the end of life. Benzodiazepine use may be associated with an increased risk of developing delirium. Alternate medications used in conjunction with benzodiazepines may serve as an independent precipitant of delirium. The aim is to understand the role of benzodiazepines in precipitating delirium and advanced mortality in palliative care population at the end of life. Methods: A retrospective medical chart review was conducted at a hospice and palliative care inpatient unit between the periods of June 2017-December 2017 and October 2017-November 2018. It included patients in hospice and palliative care inpatient units who received a benzodiazepine and those who did not. Patient characteristics, as well as Palliative Performance Scale score, diagnosis, and occurrence of admission, terminal, and/or recurrent delirium, were collected and analyzed. Results: Use of a benzodiazepine was not significantly associated with overall mortality
\end{abstract}

karger@karger.com www.karger.com/dem

Karger $\frac{1}{\%}$
(C) 2021 The Author(s)

Published by S. Karger AG, Basel

This is an Open Access article licensed under the Creative Common Attribution-NonCommercial-4.0 International License (CC BY-NC) (http://www.karger.com/Services/OpenAccessLicense), applicable to the online version of the article only. Usage and distribution for commercial purposes requires written permission. nor cause-specific death without terminal delirium rate. However, it was significantly associated with higher causespecific death with terminal delirium rate and a higher recurrent delirium rate. Discussion: This retrospective chart review suggests an association between benzodiazepine use and specific states of delirium and cause-specific death. However, it does not provide strong evidence on the use of this drug, especially at the end of life, as it pertains to the overall mortality rate. Suggested is a contextual approach to the use of benzodiazepines and the need to consider Palliative Performance Scale score and goals of care in the administration of this drug at varying periods during patient length of stay.

(C) 2021 The Author(s). Published by S. Karger AG, Basel

\section{Introduction}

Delirium is broadly defined as an acute disturbance in attention and cognition that develops over a short period of time [1]. It is the most common complication affecting hospitalized patients aged 65 years and older. It has further been shown to be associated with significant patient 
morbidity and increased mortality in several patient populations, but of particular relevance in palliative care settings where refractory delirium is common at the end of life [2]. It is noted in the literature to be prevalent in up to $42 \%$ of patients admitted to palliative care inpatient units with this proportion increasing to $88 \%$ at the end of life [1-3].

Many patients experience delirium as a state of waxing and waning altered sensorium. Accordingly, it can be difficult to discern whether a state of delirium will be reversible or not [4]. Reversibility is often emphasized given many etiologies, such as infection and medication effects, which may be treated in certain situations [5]. However, some episodes of delirium can continue for weeks, months, or even years. The persistence of delirium and repeated episodes in some patients is termed recurrent delirium and may in part be due to inadequate treatment of an underlying etiology, most often dementia $[5,6]$. Dying patients with an irreversible state of altered consciousness, frequently presenting with confusion, restlessness, and/or agitation, with or without day-night reversal, are diagnosed as having terminal delirium, where treatment is guided by patient goals of care $[4,7]$.

The pathophysiology of delirium is largely unknown; however, identified precipitating factors include medications such as opioids and benzodiazepines, infection, metabolic factors such as organ dysfunction and electrolyte disturbance, hypoxia, and dehydration [8]. Medications are increasingly recognized as a precipitant of delirium, especially in older adults and patients with altered pharmacokinetics and pharmacodynamics [9]. Accordingly, clinical recommendations include active de-prescribing of "deliriogenic" medications, of which benzodiazepines are included $[10,11]$.

A systematic review examining medication classes and delirium identified that benzodiazepine use may be associated with an increased risk of developing delirium. In particular, longer-acting benzodiazepines and higher doses within a 24 -h period worsen potentiation of delirium [12]. However, in the context of terminal delirium in which the goals of care are to maximize patient comfort, benzodiazepines are often prescribed in a concerted effort to reduce persistent restlessness and agitation $[13,14]$.

Benzodiazepines are often used as a sedative for seizures, anxiety disorders, insomnia, and agitation in the general population [15]. In palliative care, delirium is prevalent toward the end of life and can cause distress for the patients, families, and caregivers. The studies have shown that benzodiazepines can relieve symptoms of distress in this setting [16]. Midazolam and lorazepam are considered drugs of choice for delirious patients due to their rapid onset of action and short half-life [16]. Drugdrug interactions and contraindications specific for each patient should be considered when using benzodiazepines, especially in older adults. For example, midazolam is metabolized by the CYP3A4 system, and as a result, its use is contraindicated in patients with severe liver failure or in the presence of other drugs that are metabolized by the same isoform such as methadone [16]. Lorazepam is metabolized by conjugation and does not have active metabolites and as a result has more appropriate use for patients with liver damage or failure [16]. It is important to individually tailor benzodiazepines to patient's comorbidities. There is conflicting evidence on the efficacy of benzodiazepines in treating delirium.

Now more than ever, there is a rise in the development of delirium in older patients especially in those with COVID-19 symptoms. It has been suggested that delirium is one of the important symptoms in the rapid diagnosis of COVID-19 in older patients [17]. Benzodiazepines have been found to both provoke and hide the management of delirium in older adults [18]. A recent Cochrane review which examined the effect of benzodiazepines on delirium has concluded there is not enough evidence to determine whether benzodiazepines can be effective in nonICU and palliative care settings [19]. At the same time, studies showed that the addition of lorazepam especially to haloperidol may provide superior control of agitation in patients with persistent delirium with no significant differences in survival [20]. Benzodiazepines may be used in Parkinson's disease and low back pain as minimal extrapyramidal symptoms are induced when combined with antipsychotics [16]. Benzodiazepines may be used in delirium induced by sedative withdrawal, to reduce seizure risk or acute agitation crisis effective for sedation. This retrospective chart review aims to identify whether the use of benzodiazepines contributes to the development of delirium and advanced mortality in patients approaching the end of life.

\section{Materials and Methods}

To describe patients who received a benzodiazepine at the end of life and morbidity-mortality outcomes, a retrospective chart review was conducted considering patients admitted to Bethell Hospice from June 2017 to December 2017 and the palliative care inpatient unit at Baycrest from October 2017 to November 2018. Of the 92 patients identified (42 from Bethell and 50 from Baycrest), 16 patients had additional records due to one or more changes in benzodiazepine prescription during patient stay. Patients were excluded from the study if the use of a benzodiazepines was not for 
the purpose of symptom control at the end of life. Of note, Bethell was the only site to have contributed patients who were never prescribed a scheduled benzodiazepine during their length of stay.

Data were extracted from electronic medical records by 1 research assistant and included demographics such as age, sex, Palliative Performance Scale (PPS), length of stay, presence of delirium on admission, and diagnosis at both sites. The PPS is an important prognostic and clinical indicator of decline for cancer and other end-stage chronic illnesses [15]. Physical performance is measured in $10 \%$ decremental levels ranging from $100 \%$, indicating full ambulatory capacity and health, to $0 \%$, indicating death [21]. The Nurses Delirium Screening Scale (Nu-DESC) was used as a measure for delirium which consists of 5 items for rating symptoms including disorientation and inappropriate behavior [22].

For this study, a total $\mathrm{Nu}$-DESC score of $>1$ was considered a positive screen for delirium. Overall mortality was considered since all discharges were deaths, with the exception of 1 case which was transferred to a long-term care facility. Terminal delirium was recorded as "yes" or "no" for 85 of the 92 patients. Deaths with "yes" for terminal delirium were considered to be a competing risk for deaths with "no" for terminal delirium. Furthermore, any observation of delirium during the patient's length of stay was considered a delirium event, with some events being reversible and the remaining identified as irreversible terminal events. Recurrent events of delirium were identified by reviewing the Nu-DESC scores (recorded at admission, on the first day of benzodiazepine administration, 2-5 days, and $6+$ days after administration), as well as the highest Nu-DESC score and terminal delirium events. Patient length of stay was split into periods when one or more benzodiazepines were being administered and periods when no benzodiazepines were being administered.

Patients' clinical and demographic characteristics were summarized using means, standard deviations, for continuous variables and proportions for categorical data. A $t$ test was used to compare age across sites, and Fisher's exact test was used to compare sex, PPS score, presence of delirium on admission, and diagnosis across sites. Kaplan-Meier estimation was used to estimate median survival and the related 95\% confidence interval (95\% CI) for the overall mortality outcome (censored if discharged before death). The log-rank test was used to compare sites and categorized baseline PPS scores. Cox proportional hazards regression models were used to compare overall (i.e., all causes of death) mortality rates between those prescribed a benzodiazepine and those not prescribed a benzodiazepine with benzodiazepine use entered into the models as an internal time-varying covariate.

Death with and without terminal delirium were considered competing risks since terminal delirium recorded at the end of life was assumed to be irreversible, and this analysis was completed for the subset of patients with terminal delirium data available. Since prescription of benzodiazepine is a time-varying covariate, a landmark approach was used to compare the Fine-Gray subdistribution hazard rates for each risk of mortality and estimate the cumulative incidence functions [23]. Semiparametric Andersen and Gill models with robust variance estimates were used to model the outcome of recurrent events of delirium and estimate the cumulative mean function. This analysis was completed for all patients since terminal delirium data were only one source of outcome ascertainment. These models with the time-varying covariate were then further adjusted, univariately for the baseline time-invariant covari-

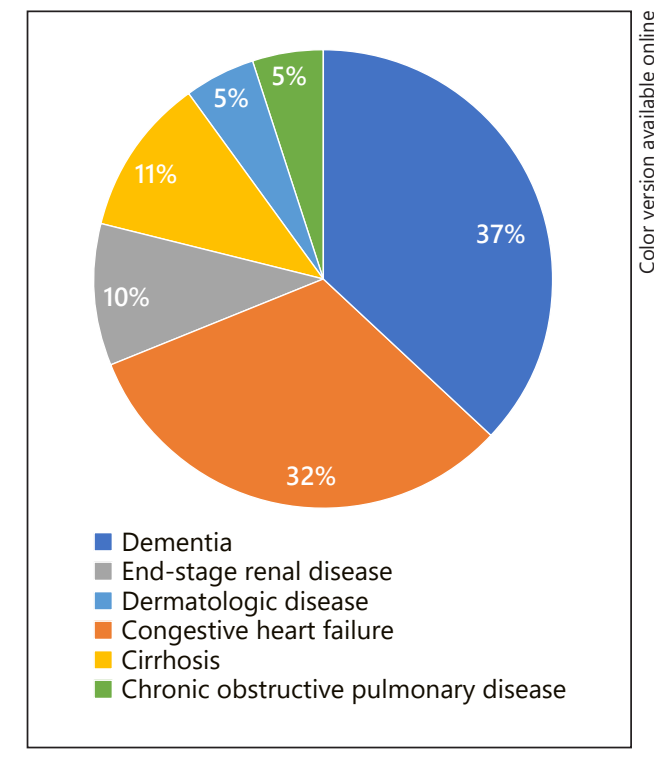

Fig. 1. Distribution of noncancer diagnoses.

ates age, sex, categorized PPS score, and diagnoses. Model estimates of hazard ratios (HR) and related $95 \%$ CIs are provided. The proportional hazards assumption for status of benzodiazepine use was tested by the interaction of this covariate with $\log$ (days). Statistical analysis was performed using SAS/STAT software version 15.2 and the SAS System for Windows version 9.4., Copyright (C) 2016 SAS Institute Inc.

\section{Results}

\section{Patient Characteristics}

The average age of patients included in this study was 76.9 ( $\mathrm{SD}=12.2)$ with both sites having similar ages $(p=$ $0.84)$. The majority of patients were female (58\%), with no significant difference in this distribution between sites $(p=0.53)$. Bethell was noted to have a higher percentage of patients with lower PPS scores, approximately $45 \%$ $(N=42)$ of whom had a score of 10-20 compared to Baycrest which had a greater percentage of patients with higher PPS scores (44\%, $N=43, \mathrm{PPS}=40, \chi^{2} p<0.0001$, $N=85)$. Primary diagnosis among patients was cancerrelated (79\%), compared to noncancer-related diagnoses (21\%). Noncancer diagnoses included dementia, congestive heart failure, end-stage renal disease, cirrhosis, dermatologic diseases, and chronic obstructive pulmonary disease (Fig. 1). Distribution of diagnoses across both sites was not significantly different $(p=0.82, N=91)$. Descriptive summaries are provided in Table 1. 
Table 1. Descriptive statistics from retrospective chart review of patients

\begin{tabular}{|c|c|c|}
\hline Patient characteristics & $\begin{array}{l}\text { Bethell hospice } \\
\text { patients, } N(\%)\end{array}$ & $\begin{array}{l}\text { Baycrest health sciences } \\
\text { patients, } N(\%)\end{array}$ \\
\hline Patients, $n$ & $42(45.6)$ & $50(54.3)$ \\
\hline Mean age (SD) & $76.6(13.1)$ & $77.1(11.4)$ \\
\hline Gender (female) & $26(61.9)$ & $27(54.0)$ \\
\hline \multicolumn{3}{|l|}{ PPS score $*$} \\
\hline PPS 10-20 & $19(45.2)$ & $3(7.0)$ \\
\hline PPS 30-35 & $19(45.2)$ & $21(48.8)$ \\
\hline PPS 40 & $4(9.5)$ & $19(44.2)$ \\
\hline \multicolumn{3}{|l|}{ Diagnosis } \\
\hline Cancer & $32(78.0)$ & $40(80.0)$ \\
\hline Noncancer & $9(22.0)$ & $10(20.0)$ \\
\hline \multicolumn{3}{|c|}{ Other medications ( $N=115$, number of available periods for 92 patients) } \\
\hline Opioid & \multicolumn{2}{|l|}{$27(23.5)$} \\
\hline Neuroleptic & \multicolumn{2}{|l|}{$4(3.5)$} \\
\hline Opioid and neuroleptic & \multicolumn{2}{|l|}{$59(51.3)$} \\
\hline Opioid, neuroleptic, and anticholinergic & \multicolumn{2}{|l|}{$20(17.4)$} \\
\hline Opioid and anticholinergic & \multicolumn{2}{|l|}{$5(4.3)$} \\
\hline
\end{tabular}

\section{Benzodiazepine Use and Other Medications}

Among the 92 patients identified, 71 (78\%) had documentation of benzodiazepine use during their length of stay. The remaining 20 patients (22\%) without documentation of scheduled benzodiazepine use were situated at the Bethell site only. For those with documentation of benzodiazepine use and a known start date $(N=68), 31$ patients (46\%) started a benzodiazepine at admission.

Of 102 records identifying patients with a prescription for a benzodiazepine, the most frequently prescribed benzodiazepines were lorazepam (52\%), clonazepam (21\%), and midazolam (15\%) with doses ranging from 0.125 to $20 \mathrm{mg}$ (Fig. 2). The majority of benzodiazepines used was scheduled (79\%) at Baycrest only and the remaining $(21 \%)$ reported at the Bethell site only. Of the identified scheduled records $(N=81)$, the majority reported a frequency of "once daily" (69\%) or twice daily (17\%).

The most frequently identified combination with alternate medication was opioids and neuroleptics (51\% of records, $N=115$ ). Opioids were used both alone or in other combinations in another $45 \%$ of the records.

\section{Overall Mortality}

All but one of the 92 palliative care patients had an outcome of death. The median time to death was 15 days (95\% CI: 11-17 days). Median length of stay was shorter at Bethell (9 days, 95\% CI: 7-17 days, $p=0.05$ ) when compared to Baycrest (17 days, 95\% CI: 13-25 days).
Baseline PPS scores were available for 85 patients. The median length of stay for patients with a PPS of 10-20 was 10.5 days (95\% CI: $7-17$ days), 14 days (95\% CI: $8-19$ days) for PPS score of 30-35, and 28 days (95\% CI: $16-38$ days) for PPS score of $40(p=0.03)$ across both sites (Fig. 3).

When considering overall mortality, that is, all causes of death, evidence for confounding between site and PPS score was identified. When comparing mortality rates for Bethell and Baycrest, unadjusted for PPS scores, statistical analysis revealed a hazard ratio (HR) of 1.6 (95\% CI: $1.1-2.6, N=85, p=0.03$ ). The direction of the HR indicates that Bethell patients had a higher overall mortality rate, related to the shorter median length of stay reported above. However, the magnitude of site-related HR was reduced and no longer significant when PPS score was adjusted for (HR 1.4, 95\% CI: $0.8-2.3, N=85, p=0.22$ ).

Overall mortality was not significantly different between those prescribed benzodiazepines compared to those who were not ( $\mathrm{HR}=1.3,95 \% \mathrm{CI}: 0.8-2.0, p=0.28$, $N=95$ ). After adjusting this model for other covariates, only the PPS score was significantly associated with overall mortality, with lower PPS scores having higher mortality rates relative to a PPS score of 40 (PPS 10-20, HR = 2.4, 95\% CI: $1.2-4.7, p=0.01$; PPS 30-35, HR 1.7, 95\% CI: $1.0-3.0, p=0.06, N=78)$.
Senderovich/Gardner/Berall/Ganion/ Zhang/Vinoraj/Waicus 


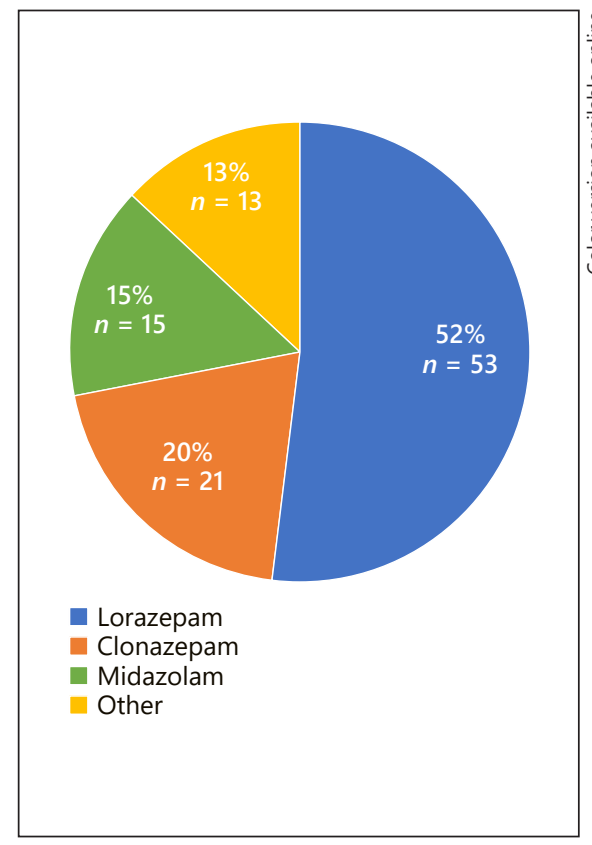

Fig. 2. Distribution of prescribed benzodiazepines.

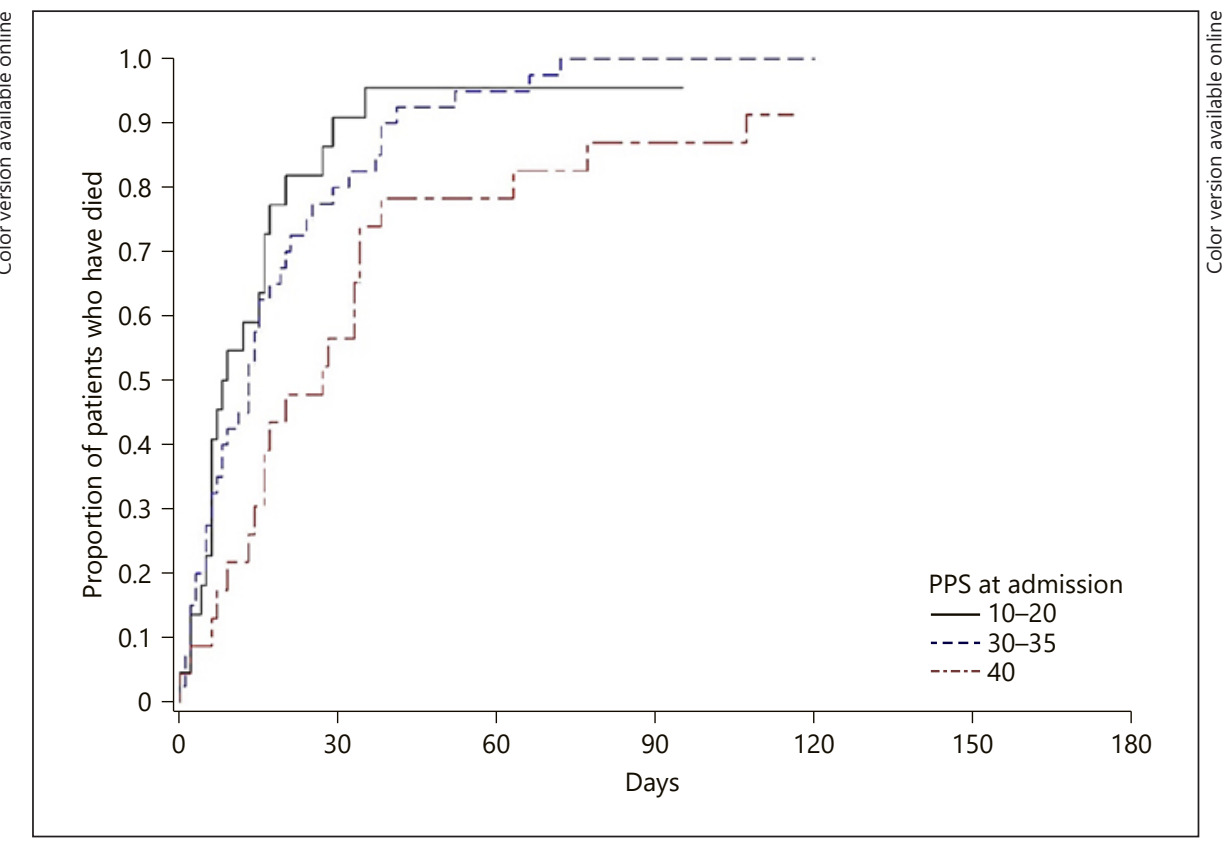

Fig. 3. Cumulative incidence functions for overall mortality by PPS at admission.

\section{Delirium}

Admission delirium was recorded for 90 of 92 patients with $20(22 \%)$ reported as "yes" and 70 (78\%) reported as "no." Admission delirium was found to be similar across both sites $(p=0.80)$. Terminal delirium was recorded for 85 of the 92 patients with 48 (56\%) reported as "yes" and 37 (44\%) reported as "no." Across all periods, 75 of the 92 patients (82\%) had 1-3 delirium events (total of 94 events), and 17 patients (18\%) had no reported delirium events during their length of stay.

\section{Competing Risks: Death with and without Terminal Delirium}

A series of models were fit at 6 landmarks $(s=0,7,14$, 21, 28, and 35 days) for each competing risk. Each model included patients with recorded terminal delirium (yes or no) who had not died as of the landmark day. Their use of benzodiazepines was fixed as of the last known value up to the landmark day. For death with terminal delirium, the hazard rate was higher when patients were prescribed a benzodiazepine compared to those who were not. The HR was significant for $s=7$ and 14 days ( $\mathrm{HR}=2.6,95 \%$ CI: $1.4-5.0, p=0.004, N=67$ and $\mathrm{HR}=2.5,95 \%$ CI: 1.2 , $5.5, p=0.02, N=48$, respectively, online suppl. Table S1; see www.karger.com/doi/10.1159/000520975 for all online suppl. material; Fig. 4) but trended toward neutral
$\mathrm{HR}=1$ by $s=35$ days. For death without terminal delirium, the hazard rate was lower when patients were prescribed a benzodiazepine compared to those who were not for most landmarks. The HR was significant for $s=0$ and 7 days $(\mathrm{HR}=0.48,95 \% \mathrm{CI}: 0.24-0.97, p=0.04, N=$ 85 , and HR $=0.44,95 \%$ CI: 0.24, 0.97, $p=0.04, N=67$, respectively, online suppl. Table S1; Fig. 4) but trended toward the neutral $\mathrm{HR}=1$ by $s=35$ days. When these models were adjusted for PPS score, the PPS score was not significantly associated with death with terminal delirium at any landmark day but was significantly associated with death without terminal delirium at each landmark day (see online suppl. Table S2). Lower PPS scores were associated with a higher rate of death without terminal delirium.

\section{Recurrent Delirium}

The recurrent delirium rate was significantly higher when patients were prescribed a benzodiazepine compared to those who were not ( $\mathrm{HR}=1.9,95 \% \mathrm{CI}: 1.2-2.9$, $p=0.007, N=92$, Fig. 5). After adjusting this model for other covariates, only the lowest PPS score category was significantly associated with recurrent delirium rate relative to a PPS score of 40 (PPS 10-20, HR 2.2, 95\% CI: $1.2-4.1, p=0.02, N=85)$. 


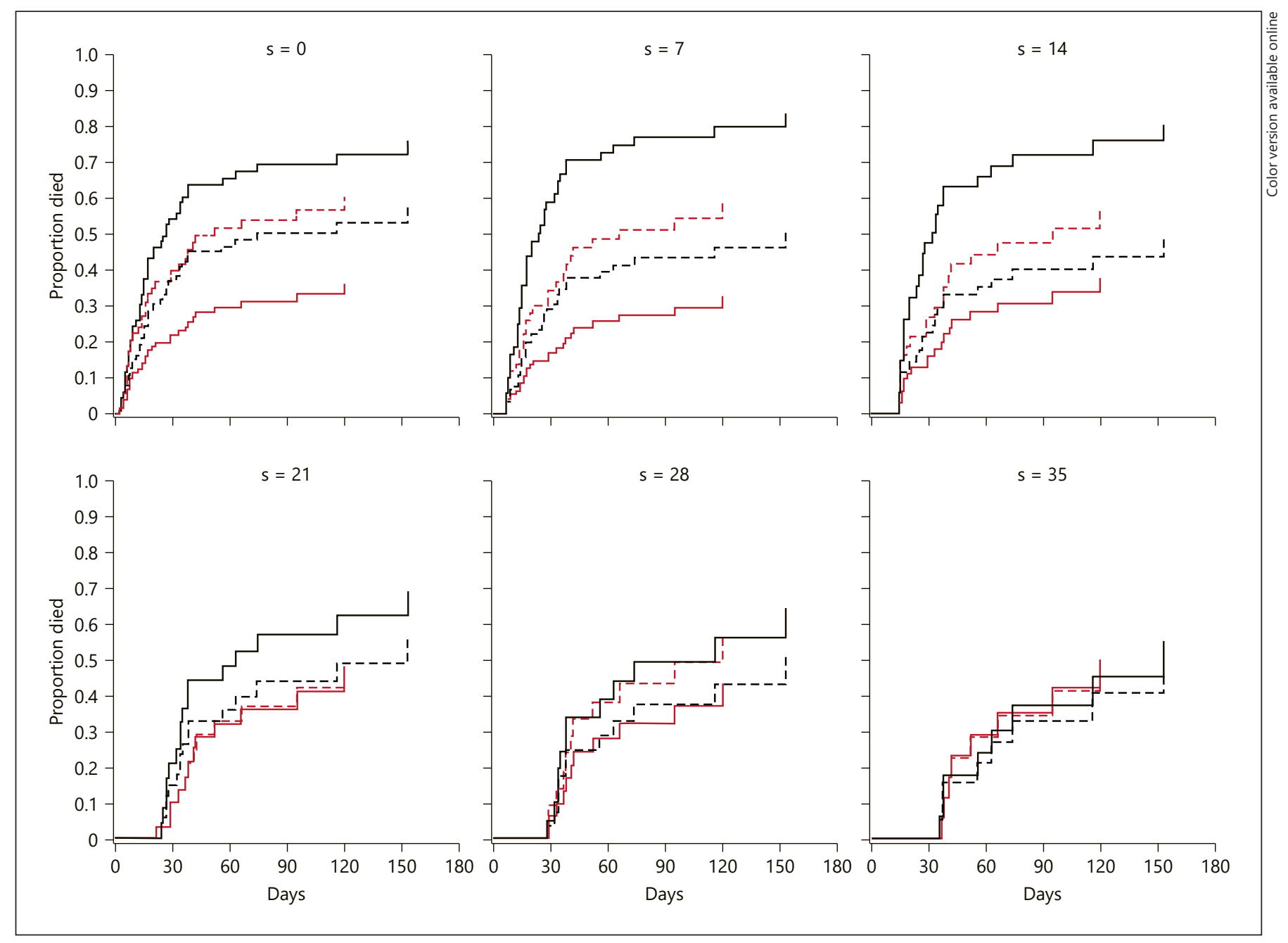

Fig. 4. Cumulative incidence functions for recurrent by use of benzodiazepine.

\section{Discussion/Conclusion}

This study examined a geriatric population at a hospice and palliative care unit where benzodiazepines were administered (continuously or periodically) over patient length of stay. Of particular focus were admission, recurrent, and terminal delirium experiences pertaining to benzodiazepine use during the patients' stay at both institutions. Patient groups between both sites had no significant differences in demographic characteristics except for baseline PPS.

Exploration of available patient medical records demonstrated that benzodiazepines were more often combined with alternate medications such as opioids and neuroleptics. Use of opioids, both alone or in combination, was noted to account for a substantial proportion of patient records which is consistent with clinical practice guidelines for symptom management at the end of life. In a study of factors associated with delirium in a frail geriatric population, patients with delirium had an average of 5.2 predisposing and 3.0 precipitating factors including the use of opioids $[19,24]$. A number of additional studies identified opioids in particular as a precipitating factor of delirium where an association was found between opioids and delirium in patients admitted to an intensive care unit and with opioid use in general [12, 25, 26].

In an additional study exploring benzodiazepine and opioid use in an older population, in patients without dementia, a statistically significant association was found between benzodiazepine-opioid use and delirium duration. In this subgroup of patients, a $142 \%$ increase in the rate of delirium among those receiving either a benzodi- 
Fig. 5. Cumulative mean function for recurrent by use of benzodiazepine.

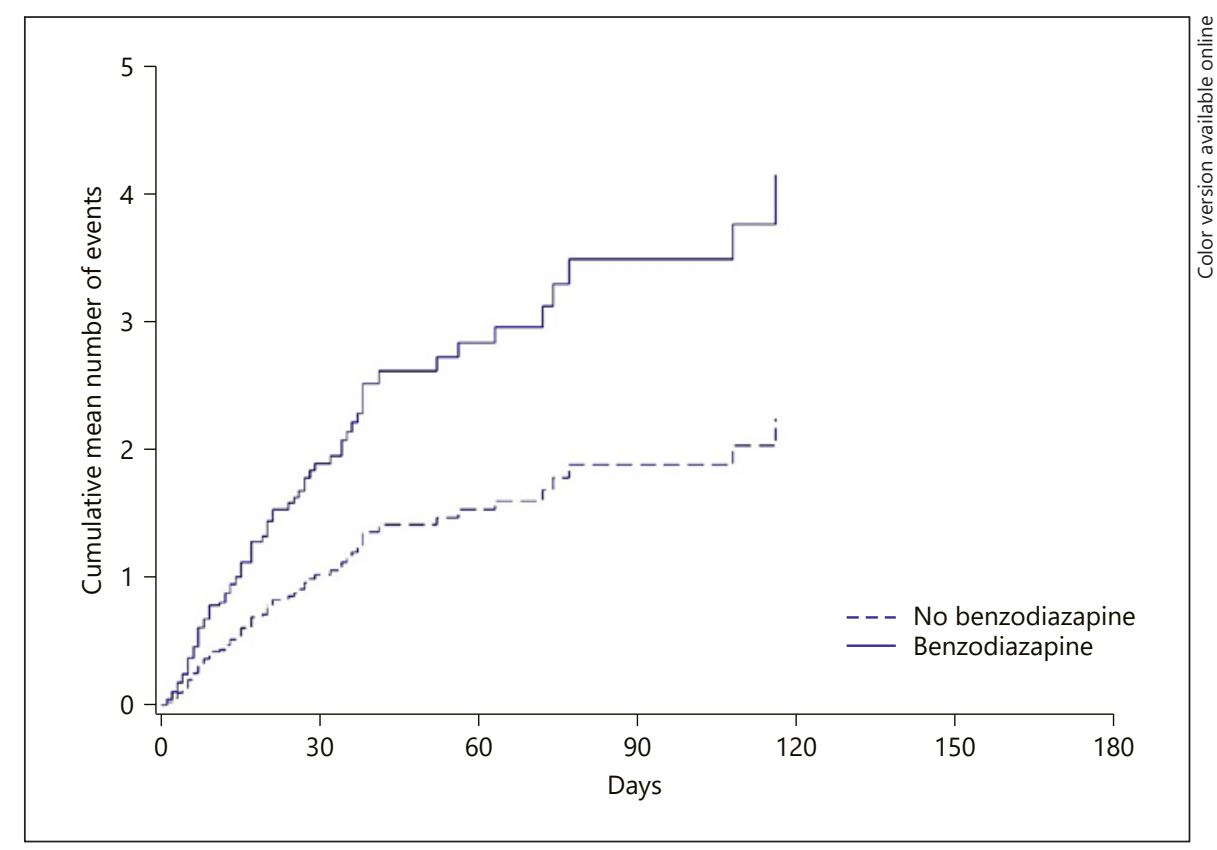

azepine or opioid was noted compared to persons not receiving this medication ( $\mathrm{RR}=2.42,95 \% \mathrm{CI}: 1.65-3.55$ ) [27]. No investigations to date have explored admission, terminal, and recurrent delirium as distinct periods or considered varying outcomes of benzodiazepine use associated with these distinct states. Our review was able to provide insight on the use of benzodiazepines and its association with higher cause-specific death with terminal delirium and higher recurrent delirium, being the first to suggest differences in outcome associated with delirium onset.

This review identified lower PPS scores to be significantly associated with higher overall mortality rate, higher rate death without terminal delirium, and higher recurrent delirium rate. This is supported in the literature considering the survival rate among those with a PPS score $>30$ compared to a score of $<30$. One study among hospitalized patients with terminal cancer identified a score change of $>30 \%$ yielded a HR for death of 2.66 (95\% CI: $2.19-3.22)$ compared to a score change of $\leq 30 \%$ [23]. A PPS score of $\leq 30$ was also independently predictive of survival with a HR of 1.67 (95\% CI: $1.30-$ 2.02) compared to a PPS score $>30$ [28]. Where our investigation noted a single PPS score at the time of admission, changes in PPS may be a more sensitive indicator of death. Decreased PPS scores are also identified in numerous studies to be associated with an increased risk of delirium [29-33].

Benzodiazepine Use in a Geriatric

Palliative Care Unit

\section{Strengths and Limitations}

Study strengths include the notation of unique delirium periods (i.e., admission, recurrent, or terminal delirium). Further to this, study samples were noted to be not significantly different between both sites, with the exception of PPS score, which may support cautious consideration of benzodiazepine use in both end-of-life care settings. The present study is limited by the extent of available data in patient electronic medical records. Although significant data cleaning was employed, uncharted information such as benzodiazepine administration date, days on benzodiazepines, and other information pertaining to medication used may have impacted the algorithm used to determine periods of benzodiazepine use within a patient's length of stay as described above. All residents present at Bethell were selected, as well as those admitted over the next 3 months (i.e., never used a benzodiazepine and PRN scheduling cases); these cases were obtained at a different time frame which may reflect differences between care providers or guidelines for the administration of benzodiazepines in these settings. Furthermore, NuDESC scores were noted to have been recorded more frequently during periods of benzodiazepine use and may therefore introduce an element of bias in the obtained results. Delineating specific criteria for the outcome measures of interest, including specifications for in-hospital/ hospice scoring of delirium, may further assist in providing robust recommendations pertaining to patient course during different periods within the length of stay. 


\section{Implications for Practice and Future Research}

While the existing body of literature notes medication combinations associated with the development of delirium, future investigations should consider specific classes of medications as independent risk factors for the potentiation of delirium. Furthermore, there is limited exploration of PPS score during varying presentations of delirium over a patient's length of stay (i.e., differentiation of PPS scores between admission, recurrent, and/or terminal delirium events). This is the only study that assessed the impact of benzodiazepines on mortality in patients with terminal and/or recurrent delirium compared to those without terminal delirium which adds further knowledge to the limited amount of existent evidence in this area and may have certain practice implications. The findings of our study suggest a need to further explore differences in morbidity-mortality outcomes between varying differing states of delirium. Clinicians treating patients with benzodiazepines should be aware of the individual risk factors and appropriate dosage for successful use of benzodiazepines, especially in the geriatric population. These findings are not limited only to palliative care. All prescribers and the public should be cognizant of the lack of evidence for benzodiazepine use in the management of symptoms. Future research that involves large studies with older patients in outpatient and inpatient settings including those who are at the end of life is warranted.

This retrospective chart review demonstrated that the use of a benzodiazepine was not significantly associated with the overall mortality rate nor the rate of death without terminal delirium. However, lower PPS scores were significantly related to a higher overall mortality rate and a higher rate of death without terminal delirium. Furthermore, use of a benzodiazepine was significantly related to a higher rate of death with terminal delirium rate and a higher recurrent delirium rate. The lowest PPS scores were significantly related to a higher recurrent delirium rate only. Although the evidence for 2 delirium outcomes (terminal and recurrent) demonstrated an increased number of cases associated with benzodiazepine use, caution is warranted in the interpretation of these results. Future investigation may help to inform benzodiazepine use at varying stages in the development of delirium at the end of life.

\section{Acknowledgments}

We would like to acknowledge Othman Farahneh and Rosanne Aleong for their participation in data collection during study development.

\section{Statement of Ethics}

Patient data were de-identified, and therefore written informed consent was not required. In addition, the study was reviewed and approved by the Baycrest Research Ethics Board on August 22, 2018.

\section{Conflict of Interest Statement}

The authors have no conflicts of interest to declare.

\section{Funding Sources}

This research did not receive any specific grant from funding agencies in the public, commercial, or not-for-profit sectors.

\section{Author Contributions}

Helen Senderovich was responsible for substantial contributions to the conception and design of the work, acquisition, analysis, and interpretation, drafting of the manuscript, critical revisions, and final approval of version to be published and accountable for all aspects of the published work. Sandra Gardner was responsible for substantial contributions in the conception and design, analysis and interpretation of data, drafting of the manuscript, and critical revisions. Anna Berall was responsible for the analysis and interpretation of data and drafting of the manuscript. Michael Gagnon was responsible for the drafting of the manuscript. Dennis Zhang was responsible for the conception and design and drafting of the manuscript. Danusha Vinoraj was responsible for drafting of the manuscript and critical revisions. Sarah Waicus was responsible for drafting of the manuscript and critical revisions.

\section{Data Availability Statement}

All data generated or analyzed during this study are included in this article and/or the online supplementary material files. Further enquiries can be directed to the corresponding author.

\section{References}

1 Hshieh TT, Inouye SK, Oh ES. Delirium in the elderly. Psychiatr Clin North Am. 2018;41(1): 1-17.

2 Bush SH, Tierney S, Lawlor PG. Clinical assessment and management of delirium in the palliative care setting. Drugs. 2017;77(15): 1623-43.

3 Hosie A, Davidson PM, Agar M, Sanderson CR, Phillips J. Delirium prevalence, incidence, and implications for screening in specialist palliative care inpatient settings: a systematic review. Palliat Med. 2013;27(6):48698. 
4 Landzaat LH, Sinclair CT. Care of the dying patient. In: McKean SC, editor. Medicine principle practice. 2nd ed. New York: McGraw-Hill; 2020. Available from: http://accessmedicine.mhmedical.com.proxy.bib.uottawa.ca/content.aspx?bookid $=1872 \&$ sectionid $=146987478$.

5 Andrew AS, Miller BL. Confusion and delirium. In: Jameson JL, editor. Harrison's principles of internal medicine. 20th ed. New York, NY: McGraw-Hill; 2021. Available from: http: //accessmedicine.mhmedical. com.proxy.bib.uottawa.ca/content. aspx?bookid $=2129 \&$ sectionid $=192011608$.

6 Chan PK. Clarifying the confusion about confusion: current practices in managing geriatric delirium. B C Med J. 2011;53(8):409-15.

7 Freemon FR. Delirium and organic psychosis. Dordrech: Springer; 1981.

8 Lawlor PG, Gagnon B, Mancini I, Pereira J, Hanson J, Suarez-Almazor M, et al. Occurrence, causes, and outcome of delirium in patients with advanced cancer: a prospective study. Arch Intern Med. 2000;160(6):86-94.

9 Lin RY, Heacock LC, Fogel JF. Drug-induced, dementia-associated and non-dementia, non-drug delirium hospitalizations in the United States, 1998-2005: an analysis of the national inpatient sample. Drugs Aging. 2010; 27(1):51-61.

10 Jackson N, Doherty J, Coulter S. Neuropsychiatric complications of commonly used palliative care drugs. Postgrad Med J. 2008; 84(989):121-5.

11 Caraceni A. Drug-associated delirium in cancer patients. EJC Suppl. 2013;11(2):233-40.

12 Clegg A, Young JB. Which medications to avoid in people at risk of delirium: a systematic review. Age Ageing. 2011;40(1):23-9.

13 Hui D. Benzodiazepines for agitation in patients with delirium: selecting the right patient, right time and right indication. Palliat Support Care. 2018;12(4):489.

14 Hui D, De La Cruz M, Bruera E. Palliative care for delirium in patients in the last weeks of life: the final frontier. J Palliat Care. 2014;30: 259-64
15 Griffin CE, Kaye AM, Bueno FR, Kaye AD. Benzodiazepine pharmacology and central nervous system-mediated effects. Ochsner J. 2013;13(2):214-23.

16 Hui D. Benzodiazepines for agitation in patients with delirium: selecting the right patient, right time and right indication. Curr Opin Support Palliat Care. 2018;12(4):489_ 94.

17 Kennedy M, Helfand BKI, Gou RY, Gartaganis SL, Webb M, Moccia JM, et al. Delirium in older patients with COVID-19 presenting to the emergency department. JAMA Netw Open. 2020;3(11):e2029540.

18 Goldberg SE, Bradshaw LE, Kearney FC, Russell C, Wittamore KH, Foster PER, et al. Care in specialist medical and medical health unit compared with standard care for older people with cognitive impairment admitted to general hospital: randomised controlled trial (NIHR TEAM trial). BMJ. 2013;347:f4132.

19 Li Y, Ma J, Jin Y, Li N, Zheng R, Mu W, et al. Benzodiazepines for treatment of patients with delirium excluding those who are cared for in an intensive care unit. Cochrane Database Syst Rev. 2020;2(2):CD012670.

20 Hui D, Frisbee-Hume S, Wilson A, Dibaj SS, Nguyen T, De La Cruz M, et al. Effect of lorazepam with haloperidol versus haloperidol alone on agitated delirium in patients with advanced cancer receiving palliative care: a randomized clinical trial. JAMA. 2017 Sep 19; 318(11): 1047

21 Victoria Hospice Society. PPS and stages; 2020. Available from: http://collaborativecurriculum.ca/en/modules/PPS/PPS-thepalliativeperformancescale-02.jsp.

22 Gaudreau JD, Gagnon P, Harel F, Tremblay A, Roy MA. Fast, systematic, and continuous delirium assessment in hospitalized patients: the nursing delirium screening scale. J Pain Symptom Manage. 2005;29(4):368-75.

23 Austin PC, Latouche A, Fine JP. A review of the use of time-varying covariates in the Fine-Gray subdistribution hazard competing risk regression model. Stat Med. 2020; 39:103-13.
24 Laurila JV, Laakkonen ML, Laurila JV, Timo SE, Tilvis RS, Reijo TS, et al. Predisposing and precipitating factors for delirium in a frail geriatric population. J Psychosom Res. 2008; 65(3):249-54.

25 Dubois MJ, Bergeron N, Dumont M, Dial S, Skrobik Y. Delirium in an intensive care unit a study of risk factors. Intensive Care Med. 2001;27(8):1297-304.

26 Marcantonio ER, Juarez G, Goldman L, Mangione CM, Ludwig LE, Lind L, et al. The relationship of postoperative delirium with psychoactive medications. JAMA. 1994;272(19): 1518-22.

27 Pisani MA, Murphy TE, Araujo KL, Slattum $\mathrm{P}$, Van Ness PH, Inouye SK. Benzodiazepine and opioid use and the duration of intensive care unit delirium in an older population. Crit Care Med. 2009;37(1):177.

28 Oh JH, Lee YJ, Seo MS, Yoon JH, Kim CM, Kang C. Change in Palliative performance scale (PPS) predicts survival in patients with terminal cancer. Korean J Hosp Palliat Care. 2017;20(4):235-41.

29 Inouye SK. Delirium in older persons. N Engl J Med. 2006;354(11):1157-65.

30 Limpawattana P, Panitchote A, Tangvoraphonkchai K, Suebsoh N, Eamma W, Chanthonglarng B, et al. Delirium in critical care: a study of incidence, prevalence, and associated factors in the tertiary care hospital of older Thai adults. Aging Ment Health. 2016;20(1): 74-80.

31 Gower LE, Gatewood MO, Kang CS. Emergency department management of delirium in the elderly. West J Emerg Med. 2012;13(2): 194-201.

32 Alagiakrishnan K, Wiens CA. An approach to drug induced delirium in the elderly. Postgrad Med J. 2004;80(945):388-93.

33 Şenel G, Uysal N, Oguz G, Kaya M, Kadioullari N, Koçak N, et al. Delirium frequency and risk factors among patients with cancer in palliative care unit. Am J Hosp Palliat Care. 2017; $34(3): 282-6$ 